\title{
Delayed reversibility of complete atrioventricular block: cardio-biliary reflex after alcohol septal ablation in a patient with hypertrophic obstructive cardiomyopathy
}

\author{
Shu Fang (1), Lan Gao, Fan Yang and Yan-jun Gong ${ }^{*}$ (])
}

\begin{abstract}
Background: Complete atrioventricular block (AVB) is a life-threatening condition that usually occurs in elderly people with organic heart disease. We herein describe a rare case of complete AVB in a young man with hypertrophic obstructive cardiomyopathy (HOCM) complicated by cholecystitis and cholangitis. Both cardio-biliary reflex and alcohol septal ablation (ASA) can cause conduction block, but the latter is often irreversible. However, their simultaneous occurrence in a patient has not been reported.
\end{abstract}

Case presentation: A 31-year-old man presented with acute cholecystitis and cholangitis and complete AVB, which had been diagnosed at a local hospital on the third day after onset. On the fourth day, he was transferred to the emergency department of our hospital because of persistent complete AVB, although his abdominal pain had been partially relieved. An echocardiogram showed a remarkably elevated left ventricular outflow tract (LVOT) gradient (105.2 $\mathrm{mmHg}$ ) despite the performance of ASA 9 years previously. The abdominal pain gradually disappeared, and normal sinus rhythm was completely recovered 11 days after onset. We determined that cardio-biliary reflex was the cause of the AVB because of the absence of other common causes. Finally, the patient underwent implantation of a permanent pacemaker to reduce the LVOT obstruction and avoid the risk of AVB recurrence.

Conclusions: Cholecystitis is a rare cause of complete AVB, which is a difficult differential diagnosis when complicated by HOCM after ASA. Clinicians should be alert to the possibility of cholecystitis in patients with abdominal pain and an unknown cause of bradycardia, complete AVB, or even sinus arrest.

Keywords: Cholecystitis, Cholangitis, Complete atrioventricular block, Cardio-biliary reflex, Alcohol septal ablation

\section{Background}

Complete atrioventricular block (AVB) is characterized as independent atrial and ventricular activity due to anatomic or functional disorders of the conduction system. Complete AVB may have physiologic, pathophysiologic, or iatrogenic etiologies, some of which are reversible [1].

*Correspondence: gongyanjun111@163.com Department of Cardiology, Peking University First Hospital, No.8 Xishiku Street, Xicheng District, Beijing 100034, China
Acute cholecystitis is a common surgical disease that is associated with arrhythmias or ST-T wave changes [2] through increased vagal tone. The latter, known as the cardio-biliary reflex, is self-restoring on an electrocardiogram (ECG) [3]. Alcohol septal ablation (ASA) was introduced in 1994 as an effective treatment option and an alternative to surgical myectomy for patients with hypertrophic obstructive cardiomyopathy (HOCM), helping to reduce left ventricular outflow tract obstruction (LVOTO) and associated symptoms. ASA-induced myocardial necrosis occurs in the basal septum, which closed 
to the cardiac conduction system and likely to cause conduction block [4].

This is the first report of 9 days of complete AVB complicated by biliary tract infection after ASA. In this mixed situation, a thorough clinical history and examination were important for accurate diagnosis and treatment.

\section{Case presentation}

A 31-year-old man presented with acute postprandial abdominal pain, vomiting, and progressive dyspnea until he was unable to walk on 5 January 2020 (Fig. 1). The patient was diagnosed with biliary tract infection and complete AVB (Fig. 2A) at a local hospital. Although his
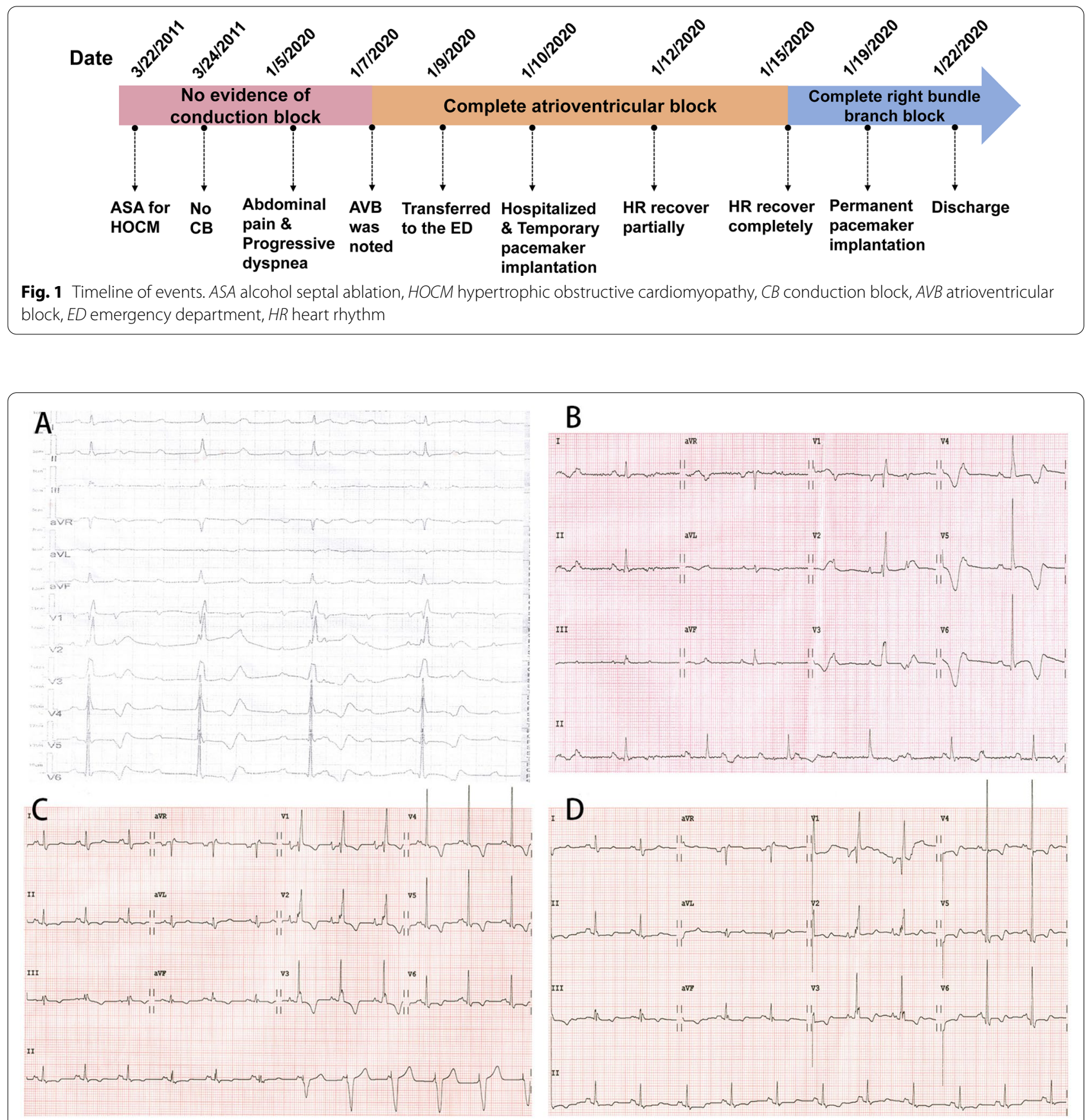

Fig. 2 Dynamic electrocardiogram changes on patient presentation. A Complete atrioventricular block (AVB) on the third day after onset in the local hospital. B Transferred to the emergency department of our hospital on the fifth day after onset. C Sinus rhythm partially passed down on eighth day after onset. D Complete right bundle branch block on 11th day after onset when AVB had disappeared 
abdominal pain was partially relieved after anti-infective therapy, the complete AVB and dyspnea remained. $\mathrm{He}$ was transferred to the emergency department of our hospital on the fourth day after onset. He was afebrile with stable hemodynamics and showed no hypoxemia. He was able to lie supine without jugular vein distention and had rales with diminished breath sounds at both lung bases. A cardiac murmur was strongest at the left margin of the sternum between the third and fourth intercostal spaces; it was a grade III/VI total systolic murmur radiating to the periphery, armpit, and back. Tenderness was present in the upper abdomen with rebound pain and muscle tension, and Murphy's sign was negative. The patient was diagnosed with HOCM in 2011 (Additional file 1: Figure S1). ASA was performed in 2011 because of recurrent syncope with a remarkably high left ventricular outflow tract (LVOT) gradient $(125 \mathrm{mmHg})$ after the Valsalva maneuver. The patient was a deliveryman without restrictions for performing heavy manual labor after ASA. He denied taking any medications.

The patient was then sent to the cardiac care unit. Laboratory tests showed a white blood cell count of $10.01 \times 10^{9} / \mathrm{L}$ (reference range, 3.5-9.5 $\times 10^{9} / \mathrm{L}$ ), neutrophil percentage of $65.7 \%$, alanine aminotransferase level of $257 \mathrm{IU} / \mathrm{L}$ (reference range, 9-50 IU/L), aspartate aminotransferase level of $110 \mathrm{IU} / \mathrm{L}$ (reference range, 15-40 IU/L), total bilirubin level of $54 \mu \mathrm{mol} / \mathrm{L}$ (reference range, $1.7-20 \mu \mathrm{mol} / \mathrm{L}$ ), brain natriuretic peptide level of $2717 \mathrm{pg} / \mathrm{mL}$ (reference range, $<100 \mathrm{pg} / \mathrm{mL}$ ), C-reactive protein level of $19 \mathrm{mg} / \mathrm{L}$ (reference range, $<5 \mathrm{mg} / \mathrm{L}$ ), and procalcitonin level of $0.18 \mathrm{ng} / \mathrm{mL}$ (reference range, $<0.05 \mathrm{ng} / \mathrm{mL}$ ). Serum electrolytes, troponin I, thyroid hormone, multiple blood cultures, antinuclear antibody, anti-dsDNA, complement $\mathrm{C} 3 / \mathrm{C} 4$, rheumatoid factor, and anti-streptolysin $\mathrm{O}$ were all normal or negative. ECG showed complete AVB with junctional escape at 40 beats/min (Fig. 2B). Chest radiographs showed no lung lesions (Additional file 2: Figure S2). Abdominal computed tomography showed cholecystitis and cholangitis with peripheral exudation without stones (Fig. 3).

Given the concern for heart failure and the previously auscultated cardiac murmur, the patient underwent echocardiographic examination. This showed mitral valve prolapse and a suspected mass, a left ventricular ejection fraction of $69.7 \%$, and LVOTO with a gradient of $105.2 \mathrm{mmHg}$ without change after the Valsalva maneuver (Fig. 4A, B). Subsequent transesophageal echocardiography revealed no valvular vegetation or mass (Fig. 4C). The patient underwent implantation of a temporary pacemaker on admission and was treated with meropenem, hepatoprotective drugs, and nutritional support.

The patient reported no abdominal pain when palpated on day 7 after onset. Unexpectedly, on day 8, we found



Fig. 3 Abdominal computed tomography. The gallbladder wall was edematous and thickened, and no gallstone was observed

that sinus rhythm could be partially passed down when attempting to reduce the pacing frequency (Fig. 2C). On the 11th day, the complete AVB disappeared, and the ECG showed complete right bundle branch block (Fig. 2D). During hospitalization, the peak velocity LVOT and LVOT gradient at rest and when provoked were monitored dynamically (Fig. 4D), and the results suggested that the LVOTO was still present even when normal rhythm was restored, either at rest or when provoked, and the pacemaker effectively reduced the LVOTO. Finally, the patient underwent permanent pacemaker implantation to reduce the LVOTO and prevent recurrence of AVB.

The patient was treated with a $\beta$-blocker and dual chamber pacing. After 18 months of follow-up, his New York Heart Association classification decreased from III to I without episodes of abnormal symptoms.

\section{Discussion and conclusions}

The causes of complete AVB are complex and various. We excluded myocardial infarction and myocarditis in this case because of the negative troponin I. Infective endocarditis could also be excluded because no valvular vegetations were present on transesophageal echocardiography. The lack of multisystem involvement made infiltrative cardiomyopathy an exception. Hyperkalemia, hyperthyroidism, and hypothyroidism could be easily ruled out based on the negative laboratory tests. In addition, AVB caused by progressive cardiac conduction system disease and cardiomyopathy are often irreversible.

The first differential diagnosis that we considered in this patient was a complication of the ASA procedure. Atrioventricular conduction disturbances following 




Fig. 4 Echocardiogram. A Parasternal long-axis view on admission. B Doppler ultrasonic spectrum of LVOT on admission. CTransesophageal echocardiograph showed mitral valve prolapse and no vegetation. D Dynamic change of LVOT gradient and peak velocity. This patient underwent alcohol septal ablation on March 22, 2011 and permanent pacemaker on January 19, 2020. LVOT left ventricular outflow tract, ASA alcohol septal ablation, NM not measured, AVB atrioventricular block, TPM temporary pacemaker, PM pacemaker, PPM permanent pacemaker

ASA are mainly seen in elderly patients, and pacemaker implantation is needed in only $5 \%$ of young patients $[5$, 6]. Patients with left bundle branch block, a P-R interval of $>200 \mathrm{~ms}$, a greater pressure gradient during exercise before ablation, and advanced age are more likely to develop AVB after ASA [7, 8]. Complete AVB after ASA is usually seen within the first $24 \mathrm{~h}$ [9]. In one study, the incidence of late complete AVB was $8.9 \%$ more than $48 \mathrm{~h}$ after ASA and only $3.6 \%$ from 4 days to 3 years after ASA; no cases of complete AVB occurred after 3 years [8]. Late complete AVB may be explained by myocardial scarring and fibrosis after ASA $[8,10]$, and these changes are progressive and unlikely recoverable. Our patient had few risk factors for AVB, and no AVB was found within $48 \mathrm{~h}$ after ablation (Additional file 3: Figure S3). Complete AVB can aggravate LVOTO, which in turn leads to acute heart failure [11]. However, our patient had no typical symptoms of heart failure or episodes of syncope in daily life with heavy manual labor after ASA until the onset of acute biliary tract infection. Therefore, ASA was excluded as the cause of AVB, and the cardio-biliary reflex was determined to be the rare cause of the reversible AVB.

The cardio-biliary reflex is triggered by increased tension in the gallbladder via autonomic vagal innervations in patient with cholecystitis, regardless of the presence of pain or calculi $[12,13]$. Bradycardia is one of the clinical presentations and is termed "Cope's sign"; this phenomenon was first documented in 1971 by O’Reilly and Krauthamer [3]. The cardio-biliary reflex also mimics the special ECG changes of acute coronary syndrome, such as ST-segment elevation and T-wave inversion, which are identified and treated by atropine $[2,13,14]$. The cardio-biliary reflex is often a diagnosis of exclusion. Because of the lack of evidence of other diseases in this case, we speculated the cardio-biliary reflex was the predominant cause of the complete AVB. As shown in Table 1, only five such cases of conduction block have been reported, and the longest duration was about $48 \mathrm{~h}$ [12, 15-18]. Similar to the case reported by Lau et al. [12], our patient had persistent AVB despite disappearance of his abdominal pain, indicating that the inflammatory response may have resulted in prolonged activation of the cardio-biliary reflex although his inflammatory markers were slightly raised. Notably, complete right bundle branch block may have been involved in the unusually long duration of complete AVB, which was not mentioned in any previous cases.

According to the European Society of Cardiology guidelines on cardiac pacing and cardiac resynchronization therapy, permanent pacemaker implantation can be completely avoided if the conduction block is curable and self-recoverable [19]. Pacemaker therapy is not as effective as surgery or alcohol septal reduction in reducing the gradient; older patients are more likely to benefit from these procedures, and septal myectomy 


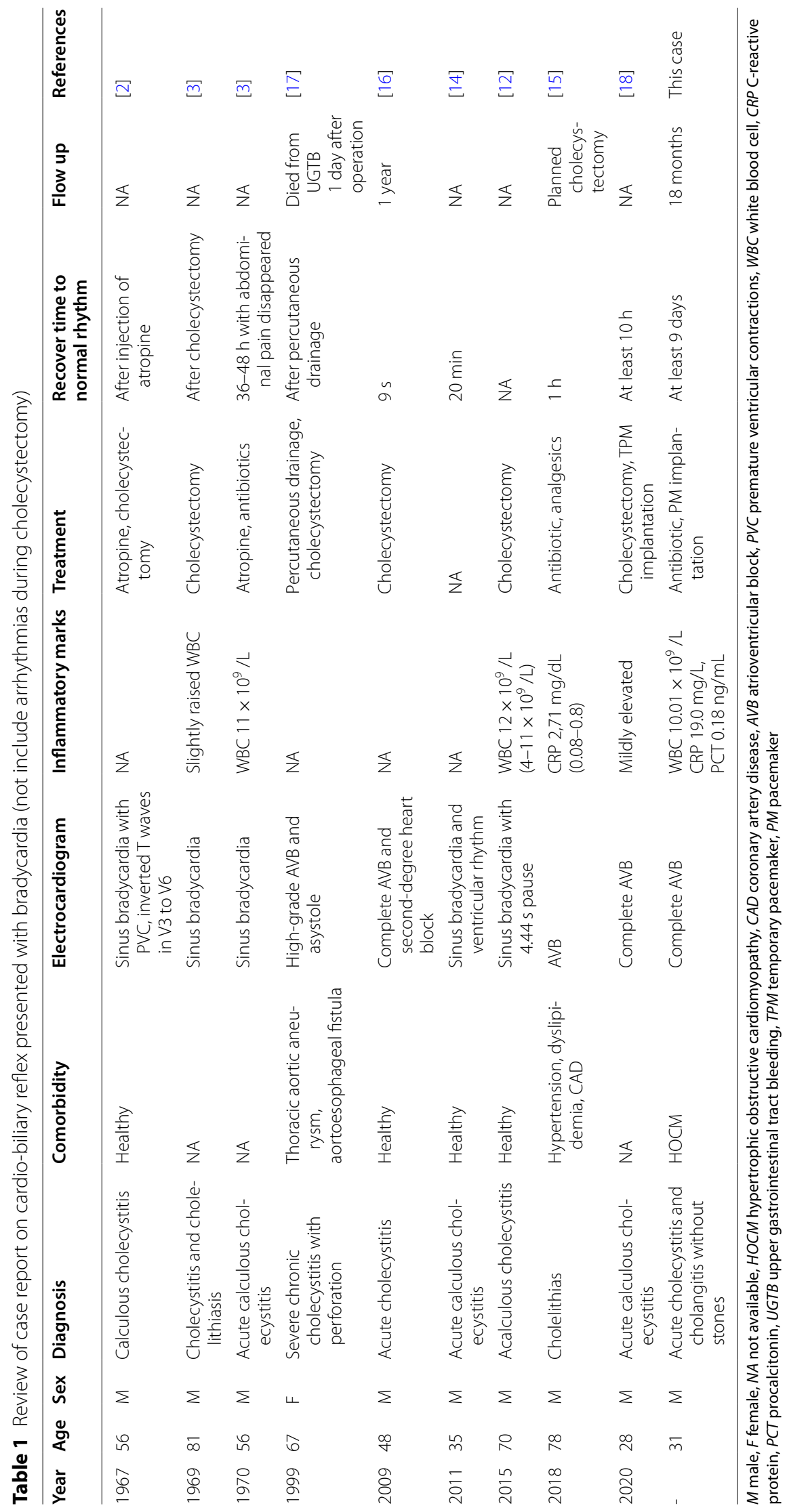


is recommended if mitral valve repair is required for treatment of mitral valve prolapse [11]. Our patient refused cholecystectomy and septal myectomy plus mitral valve prolapse repair surgery. After a multidisciplinary discussion, permanent pacemaker implantation was recommended based on the following three considerations: first, the cholecystitis may recur, which may cause recurrence of the AVB; second, a permanent pacemaker may reduce the LVOTO; and third, a pacemaker may provide security for the use of a $\beta$-blocker.

This case revealed a rare etiology and the longest duration (at least 9 days) of complete AVB complicated by acute biliary tract infection and HOCM after ASA. Cholecystitis causes multiple ECG abnormalities, and close monitoring and management are necessary to avoid misdiagnosis and possible complications. Further research is needed to elucidate the mechanism of the cardio-biliary reflex.

\section{Abbreviations}

ASA: Alcohol septal ablation; HOCM: Hypertrophic obstructive cardiomyopathy; AVB: Atrioventricular block; ECG: Electrocardiogram; LVOT: Left ventricular outflow tract; LVOTO: Left ventricular outflow tract obstruction.

\section{Supplementary Information}

The online version contains supplementary material available at https://doi. org/10.1186/s12872-021-02165-5.

Additional file 1: Figure S1. Cardiac magnetic resonance imaging.

Additional file 2: Figure S2. Chest radiograph.

Additional file 3: Figure S3. Electrocardiogram in 2011.

\section{Acknowledgements}

The authors thank the patient and his family for granting their permission to publish this case report. The authors also thank Angela Morben, DVM, ELS, from Liwen Bianji (Edanz) (www.liwenbianji.cn/ac), for editing the English text of a draft of this manuscript.

\section{Authors' contributions}

FS, GL, and YF diagnosed and treated the patient. FS reviewed the literature and drafted the manuscript. GL and YF reviewed the literature. GYJ revised the manuscript for important intellectual content. All authors issued final approval for the version to be submitted. All authors read and approved the final manuscript.

\section{Funding}

None.

\section{Availability of data and materials}

Data are available from the corresponding author upon reasonable request.

\section{Declarations}

Ethics approval and consent to participate

Not applicable. Study approval was not necessary because this is a case report, and consent for publication was obtained from the patient.

\section{Consent for publication}

Written informed consent was obtained from the patient for the publication of this case report. A copy of the written consent is available for review by the Editor-in-Chief of this journal.

\section{Competing interests}

The authors declare that they have no competing interests.

Received: 12 March 2021 Accepted: 15 July 2021

Published online: 03 August 2021

References

1. Sodeck GH, Domanovits H, Meron G, Rauscha F, Losert H, Thalmann M, Vlcek M, Laggner AN. Compromising bradycardia: management in the emergency department. Resuscitation. 2007;73(1):96-102.

2. Kaufman JM, Lubera R. Preoperative use of atropine and electrocardiographic changes. Differentiation of ischemic from biliary-induced abnormalities. JAMA. 1967;200(3):197-200.

3. O'Reilly MV, Krauthamer MJ. "Cope's sign" and reflex bradycardia in two patients with cholecystitis. Br Med J. 1971;2(5754):146.

4. Lawrenz T, Lieder F, Bartelsmeier M, Leuner C, Borchert B, Meyer Z, Vilsendorf D, Strunk-Mueller C, Reinhardt J, Feuchtl A, Stellbrink C, et al. Predictors of complete heart block after transcoronary ablation of septal hypertrophy: results of a prospective electrophysiological investigation in 172 patients with hypertrophic obstructive cardiomyopathy. J Am Coll Cardiol. 2007;49(24):2356-63.

5. Leonardi RA, Townsend JC, Patel CA, Wolf BJ, Todoran TM, Powers ER, Steinberg DH, Fernandes VL, Nielsen CD. Alcohol septal ablation for obstructive hypertrophic cardiomyopathy: outcomes in young, middleaged, and elderly patients. Catheter Cardiovasc Interv. 2013;82(5):838-45.

6. Liebregts $M$, Steggerda RC, Vriesendorp PA, van Velzen $H$, Schinkel AF, Willems R, van Cleemput J, van den Berg MP, Michels M, ten Berg JM. Long-term outcome of alcohol septal ablation for obstructive hypertrophic cardiomyopathy in the young and the elderly. JACC CardiovasC Interv. 2016;9(5):463-9.

7. Axelsson A, Weibring K, Havndrup $O$, Kelbaek $H$, Jorgensen $E$, Helqvist $S$, Iversen K, Kober L, Bundgaard H, Jensen MK. Atrioventricular conduction after alcohol septal ablation for obstructive hypertrophic cardiomyopathy. J Cardiovasc Med (Hagerstown). 2014;15(3):214-21.

8. Schuller JL, Zipse MM, Krantz MJ, Blaker B, Salcedo E, Groves BM, Messenger JC, Beaty B, Sauer WH. Incidence and predictors of late complete heart block after alcohol septal ablation treatment of hypertrophic obstructive cardiomyopathy. J Interv Cardiol. 2015;28(1):90-7.

9. Khouzam RN, Naidu SS. Current status and future perspectives on alcohol septal ablation for hypertrophic obstructive cardiomyopathy. Curr Cardiol Rep. 2014;16(5):478.

10. Raute-Kreinsen U. Morphology of necrosis and repair after transcoronary ethanol ablation of septal hypertrophy. Pathol Res Pract. 2003;199(3):121-7.

11. Elliott PM, Anastasakis A, Borger MA, Borggrefe M, Cecchi F, Charron P, Hagege AA, Lafont A, Limongelli G, Mahrholdt H, et al. 2014 ESC Guidelines on diagnosis and management of hypertrophic cardiomyopathy: the Task Force for the Diagnosis and Management of Hypertrophic Cardiomyopathy of the European Society of Cardiology (ESC). Eur Heart J. 2014:35(39):2733-79

12. Lau YM, Hui WM, Lau CP. Asystole complicating acalculous cholecystitis, the "Cope's sign" revisited. Int J Cardiol. 2015;182:447-8.

13. Krasna MJ, Flancbaum L. Electrocardiographic changes in cardiac patients with acute gallbladder disease. Am Surg. 1986;52(10):541-3.

14. Akyel A, Yayla C, Kunak T, Yaman B, Tavil Y, Boyaci B. Idioventricular rhythm in a patient with acute cholecystitis. Clin Res Hepatol Gastroenterol. 2011:35(11):774-6.

15. Papakonstantinou PE, Asimakopoulou NI, Kanoupakis E, Maragkoudakis S, Panagiotakis S, Gikas A. Cope's sign and complete heart block in a 78-year-old patient with biliary colic. Int Emerg Nurs. 2018;37:3-5.

16. Franzen D, Jung $S$, Fatio $R$, Brunckhorst CB. Complete atrioventricular block in a patient with acute cholecystitis: a case of cardio-biliary reflex? Eur J Emerg Med. 2009;16(6):346-7. 
17. Vloka ME, Tamis JE, Steinberg JS. Neurally mediated syncope in 2 patients with extracardiac disease. Arch Intern Med. 1999;159(6):625-7.

18. Ola RK, Sahu I, Ruhela M, Bhargava S. Cope's sign: a lesson for novice physicians. J Family Med Primary Care. 2020;9(10):5375-7.

19. Brignole $M$, Auricchio A, Baron-Esquivias $G$, Bordachar P, Boriani $G$, Breithardt OA, Cleland J, Deharo JC, Delgado V, Elliott PM, et al. ESC Guidelines on cardiac pacing and cardiac resynchronization therapy: the Task Force on cardiac pacing and resynchronization therapy of the European Society of Cardiology (ESC). Developed in collaboration with the European Heart Rhythm Association (EHRA). Eur Heart J. 2013;34(29):2281-329.

\section{Publisher's Note}

Springer Nature remains neutral with regard to jurisdictional claims in published maps and institutional affiliations.
Ready to submit your research? Choose BMC and benefit from:

- fast, convenient online submission

- thorough peer review by experienced researchers in your field

- rapid publication on acceptance

- support for research data, including large and complex data types

- gold Open Access which fosters wider collaboration and increased citations

- maximum visibility for your research: over 100M website views per year

At BMC, research is always in progress.

Learn more biomedcentral.com/submissions 